\title{
Optimal Parameter Tuning of MRPID Controller for Temperature Control
}

\author{
Abhas Kanungo, Monika Mittal, Lillie Dewan
}

\begin{abstract}
This paper presents the optimal tuning of gain parameters of Multi-resolution PID controller for thermal system. Control of temperature in thermal system is very important. MRPID controller utilizes the multi-resolution property of wavelet transform to decompose the error signal in to different frequency components. Further different coefficients of wavelet are used to generate the control signal. To generate the desired control, optimal tuning is required. In this paper, optimal tuning of MRPID controller is done by genetic algorithm (GA) \& Particle swarm optimization (PSO). At the end, performance comparison between these two techniques is done and concluded. Wavelet-based MRPID controller is executed in MATLAB/Simulink@2015a.
\end{abstract}

Index Terms: Thermal system, MRPID Controller, GA and PSO, MWT

\section{INTRODUCTION}

Thermal system is very important part of power industry [1]. Temperature regulation in thermal system is very much required to get optimal performance of the system [2]. PID controller has been a favorite choice in power industry [3]. Structure of PID controller is very simple [4]. Zeigler-Nichols method is very traditional method which is used to tune PID controller $[5,6]$. Comparison of Haar and Daubachies wavelet denoising techniques was done by the present authors that shows performance of PID controller is affected in presence of noise. Also, MATLAB wavelet toolbox was used for the study limiting the flexibility especially for higher order systems. Hence, there is a need of advanced controller to compensate for the disturbances [7]. Intelligent control techniques are better choice to overcome disadvantages of PID controller. Fuzzy, and neural network approaches can be utilized to tune PID controller [8]. But it increases the complexity of system. Also these are very sensitive to disturbances [9]. To overcome disturbance uncertainty, MRPID controller has been developed [10, 11]. Nowadays MRPID controller has been used in thermal system [12]. It utilizes multi resolution property of wavelet to decompose the error signal in to different frequency components [13, 14]. That property of wavelet is known as multi wavelet transform (MWT). The biggest advantage of MRPID controller is that it is very effective in presence of noise [15]. But to get optimal performance of MRPID controller proper tuning is required. In this paper GA and PSO optimization techniques have been used with wavelet based MRPID controller and concluded.

Revised Manuscript Received on June 15, 2019.

Abhas Kanungo, Electronics and Instrumentation Deptt., KIET group of institutions, Ghaziabad, India.

Monika Mittal, Electrical Engineering Deptt., NIT Kurukshetra ,India.

Lillie Dewan, Electrical Engineering Deptt., NIT Kurukshetra, India.
To achieve optimal performance of thermal system GA and PSO based optimization techniques have been used with wavelet based MRPID controller. Thermal control system is explained in section 2. Proposed MRPID controller either optimization techniques are explained in section 3. Finally, simulated results have been explained followed by conclusion at the end.

\section{THERMAL CONTROL SYSTEM}

In this paper, single input - single output system has been considered. In this system air is sucked in through surrounding air that will flow through air flow pipe. Bead thermistor is used to measure the temperature of outgoing error. To get an optimal performance of system difference between reference temperature and measured temperature should be zero. Error signal has been decomposed and used to generate the control signal with the help of MRPID controller. The control signal is given to the thermal system which further takes necessary action on the system. Optimization of MRPID controller has been done by optimization techniques (GA and PSO). The schematic diagram of thermal control system has been presented in Fig. 1[16,17].

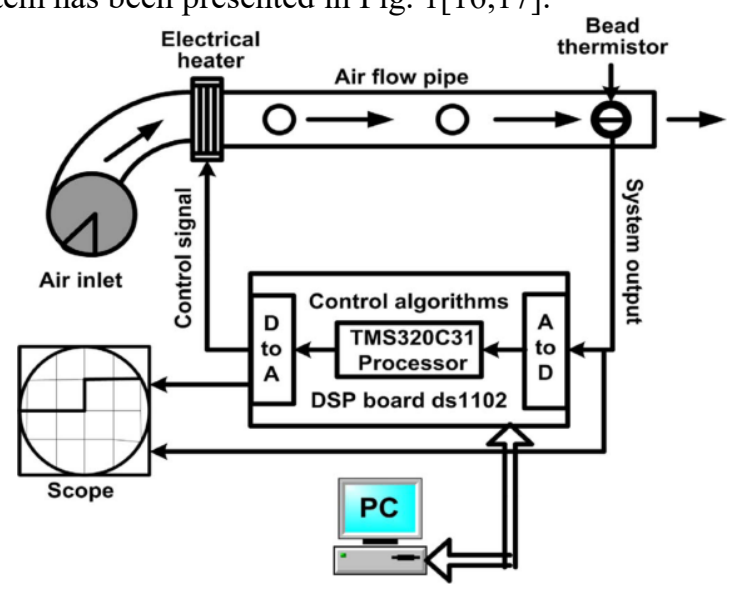

Fig1: Thermal control System

The input output relationship of thermal system is given by recognized transfer function as in equation (1),

$$
G(s)=\frac{(0.0882 s+1.26) e^{-s T d}}{0.00073 s^{3}+0.0153 s^{2}+0.585 s+1}
$$

Here $T_{d}$ is denoted as time delay.

\section{PROPOSED METHODOLOGY}

This part describes the proposed optimization techniques and MRPID controller. In this paper two 


\section{Optimal Parameter Tuning of MRPID Controller for Temperature Control}

optimization techniques have been used with MRPID controller. Multiresolution property of wavelet has been utilized to decompose the error signal which is estimated from reference and measured value of temperature. The closed loop diagram of thermal control system with MRPID controller has been shown in figure 1 .

\section{A. MRPID Controller}

Multiresolution property of wavelet has been utilized to decompose the error signal in various compact waves known as wavelets. Wavelet transform gives higher advantage than the customary PID controller in terms of compensating the error [18]. So, control output of PID controller combining three terms $K_{p}, K_{i}$ and $K_{d}$ (proportional, derivative and integral gains) is given in equation (2)-

$u_{P 1 D}=K_{p} e+K_{i} \int e d t+K_{d} \frac{d}{d t} e$

In frequency data, $\mathrm{K}_{\mathrm{p}}$ and $\mathrm{K}_{\mathrm{I}}$ terms are utilized to catch low frequency data and high frequency data of uncertainty respectively. Similarly, an MRPID decomposes the error signal into high, low and intermediate scale components are described in equation (3)-

$f(x)=f_{H}(x)+f_{M 1}(x)+\cdots+f_{M(n-1)}(x)+f_{L}(x)$

Each component is scaled by separate gains and included to create controller output signal $u$ or relating wavelet decomposition to the error signal just as aggregate impact of numerous fundamental wonders [16]. Every component is scaled by individual gains at that point included to produce control signal $(\mathrm{u})$ is given as condition (4)-

$u_{M R P I D}=K_{H} e_{H}+K_{M} e_{M 1}+\cdots+K_{M(n-1)} e_{M(n-1)}+K_{L} e_{L}$ (4)

Figure 2 represents the schematic diagram of wavelet-based self-tuning MRPID controller.

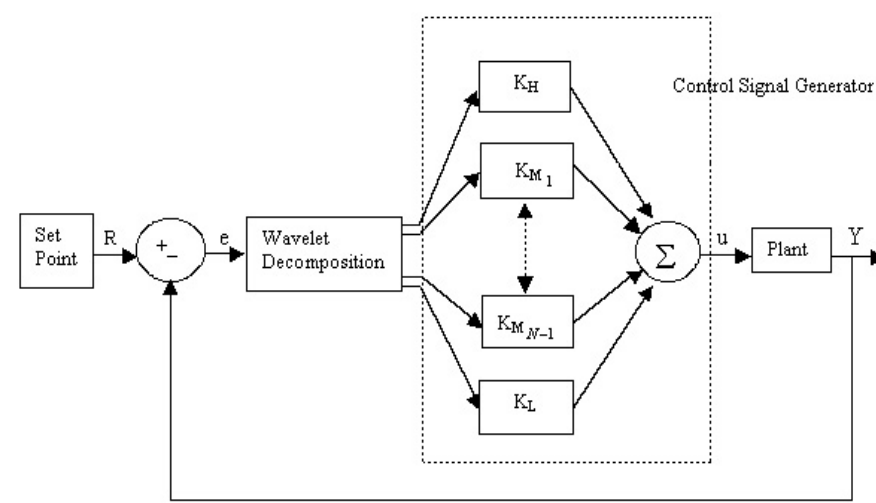

Fig2: Schematic diagram of self tuning MRPID controller

A MRPID controller disintegrates temperature uncertainty into its detail (high-recurrence) and estimation (low-recurrence) segments. A suggested MRPID controller is combination of three parameters that is $\mathrm{K}_{\mathrm{H}}, \mathrm{K}_{\mathrm{M}}$ and $\mathrm{K}_{\mathrm{L}}$. Decomposition of error signal is done up to the $2^{\text {nd }}$ level of resolution of MWT. As mentioned earlier gain parameters $\mathrm{K}_{\mathrm{H}}$ and $\mathrm{K}_{\mathrm{M}}$ are used to tune high frequency component and medium frequency components respectively and gain $\mathrm{K}_{\mathrm{L}}$ is used for low frequency component.

\section{B. GA optimization}

GA depends on set of universal solution called as global population information. In GA fitness function is define according to the specified problem. Then the selection of best solution takes place by combining the selected solution or parent classes through which child classes or new solutions have been found. It is done by the genetic operator crossover and mutation. This process will be carry on till the optimal solution of specified problem is achieved according to the fitness function [19]. Various steps involved in Genetic algorithm is explained below-

$>$ Initial Population

$>$ Fitness test by defining fitness function

$>$ Select best parent

$>$ Breeding by genetic operators

$>$ New child classes have been obtained

$>$ Mutate the child classes

$>$ Replace initial population by new population

$>$ Again go for fitness test

This process will be repeated until optimal solution of problem has been reached.

\section{GA optimization with MRPID controller}

Optimization of MRPID controller with GA can be done using following steps-

1. Initial population of size $\mathrm{N}$ is generated randomly, which generally contains binary strings.

2. The desired objective function which gives best transient characteristics like small overshoot, speedy rise time, smallest settling time is defined.

3. Each chromosome of initial population is tested by fitness function and assigned a fitness value to create new child class population by using genetic operators like crossover, mutation and reproduction.

4. Each of the chromosome consists of separate strings of MRPID term $\left(\mathrm{K}_{\mathrm{H}}, \mathrm{K}_{\mathrm{L}}, \mathrm{K}_{\mathrm{M}}\right)$

5. There are number of methods that can be used for fitting chromosome to larger probability. Roulette wheel method has been used in this paper.

6 . The ultimate objective is to reduce the error and obtain desired time domain characteristics.

7. Steps 1-6 are repeated till optimum gain values are obtained.

\section{PSO optimization}

PSO is a computational method to optimize the given problem by iteratively to improve a candidate solution with respect to a given measure of quality. It is stochastic evolutionary computation optimization technique. But unlike GA, PSO do not use evolution operators like crossover and mutation. In PSO potential solutions are called as particles. Each of the particle keeps the track of its coordinates in problem space, which are associated with best solutions with respect to the specified problem. The velocity of each particle is changed on the basis of Pbest and Ibest locations. The stored fitness value is called as Pbest and best value which is obtained by PSO optimizer is known as Ibest. Gain parameter optimization by PSO technique is referred form reference [20, 21]. Various steps involved in PSO optimization is explained below-

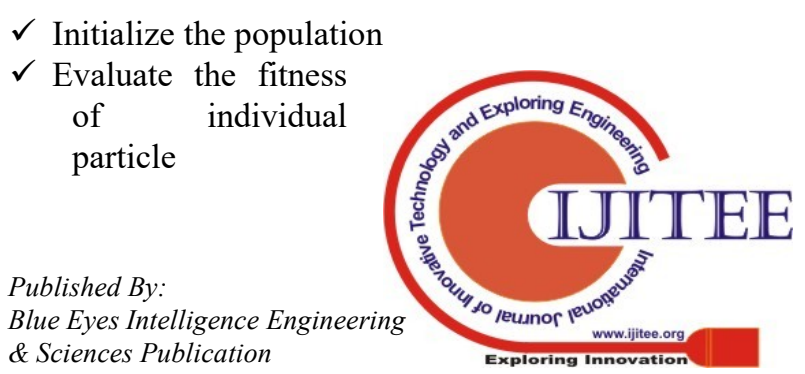


$\checkmark$ Modify velocities of previous best and global best solutions

$\checkmark$ Terminate if condition is reached

$\checkmark$ If not go to step 2

\section{PSO optimization with MRPID controller}

The first step in PSO optimization is its initialization to find the number of iterations. It is mandatory to define the upper and lower bound of three gain parameters of controller and initialize the population with searching point. Then for each initial individual, performance criteria for time domain characteristics is calculated Evaluation value is computed afterwards using evaluation function. Comparison of each evaluation value is then done with its pbest value and best value is termed as ibest. Modification in member velocity and position of each individual is undertaken till the end of the iterations. Last updated ibest value is used for optimal controller parameter.

\section{RESULT AND DISCUSSION}

In this section, analysis of thermal system with proposed optimization of a MRPID controller has been discussed. GA and PSO optimization techniques have been used to optimize the gain values of MRPID controller. Simulated results are evaluated with the help of MATLAB@2015a software. Reference value of temperature is taken as $268^{\circ} \mathrm{C}$. The comparative results of two optimization techniques in stable input conditions has been shown in figure 3 .

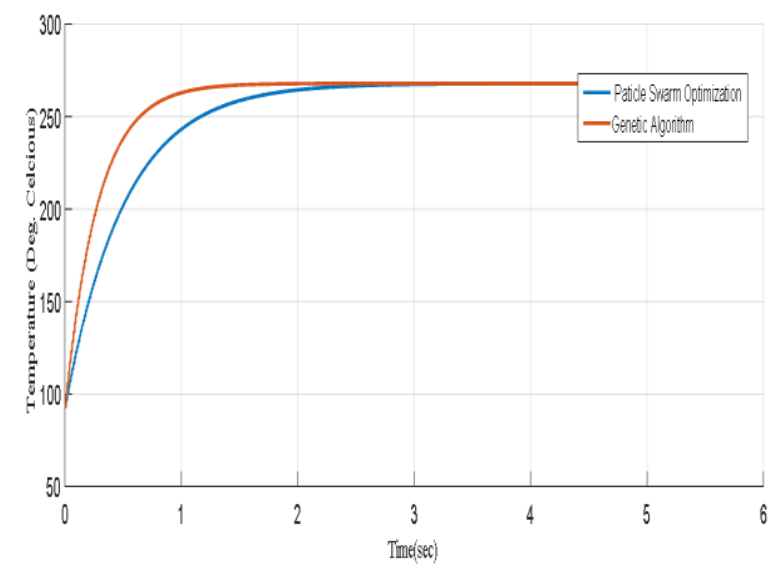

Fig.3: Comparative analysis of GA and PSO

In closed loop system, the output takes certain time to adapt for every change in the input. This part of the response is called transient phase. The time required by the output to settle to the desired value finally is known as settling time. This part of the response is known as steady state. Transient response should be more vertical for fast system response, which is required for agile systems such as the present thermal system. The required temperature should be quickly attained in this thermal system to avoid damage. It can be clearly seen in obtained result that GA optimization technique performs better in terms of transient and steady state characteristics as compared to PSO technique.

\section{CONCLUSION}

To get desired performance of MRPID controller optimal tuning is required. In this paper, GA and PSO optimization techniques have been utilized successfully to get desired output of thermal system. Multiresolution property of wavelet has been used to decompose the error signal. Further optimization of gain values takes place to generate the control signal for thermal system. In future, hybrid optimization techniques may be used to get better performance of thermal system. Also, property of MRPID controller may be utilized for different systems.

\section{REFERENCES}

1. Jino, Y., Matsumoto, S., \& Kamito, A. "Risk based Model Predictive Control with hybrid system structure and its application to Thermal Power Plants". 2006 SICE-ICASE International Joint Conference.doi:10.1109/sice.2006.315571

2. Emami-Naeini, A., Ebert, J. L., de Roover, D., Kosut, R. L., Dettori, M. Porter, L. M. L., \& Ghosal, S.”Modeling and control of distributed thermal systems". IEEE Transactions on Control Systems Technology, 11(5), 668-683.doi:10.1109/tcst. 2003.816411.

3. Dong, Z., Su, Y., \& Yan, X. "Temperature Control System of the Thermal Analyzer Based on Fuzzy PID Controller". 2009 Ninth International Conference on Hybrid Intelligent Systems.doi:10.1109/his.2009.123

4. Dong, Z., Su, Y., \& Yan, X. "Temperature Control System of the Thermal Analyzer Based on Fuzzy PID Controller". Ninth International Conference on Hybrid Intelligent Systems .doi:10.1109/his.2009.123.

5. C. C. Hang, K. J. Aström, and W. K. Ho, "Refinements of the ZieglerNichols tuning formula", Proc. Inst. Elect. Eng.-Control Theory Appl., vol. 138 , no. 2 , pp. 111-118,1991

6. J. G. Ziegler and N. B. Nichols, "Optimal settings for automatic controllers, Trans. ASME”, vol. 64, no. 11, pp. 759-768,1942.

7. Abhas Kanungo, Monika Mittal, Lillie Dewan , "Comparison of Haar and Daubechies Wavelet Denoising for PID Controlled Thermal System", Journal of Advanced Research in Dynamical and Control Systems, 2018, issue 9, pp 2405-2411.

8. Tang, K. S., Kim Fung Man, Guanrong Chen, \& Kwong, S. “An optimal fuzzy PID controller". IEEE Transactions on Industrial Electronics, 48(4), 2001,757-765. doi:10.1109/41.937407.

9. Hari Om Bansal, Rajamayyoor Sharma, P. R. Shreeraman , “ PID Controller Tuning Techniques: A Review", Journal of Control Engineering and Technology (JCET) ,2012,Vol. 2 Iss. PP. 168-176.

10. Khan, M.A.S.K. and M.Azizur Rahman, "Implementation of wavelet-based controller for battery storage system of hybrid electric vehicles", IEEE Transactions on Industry Applications, 2011,Vol.47, No.5, pp.2241-2249.

11. Das, P., Edavoor, P. J., Raveendran, S., Rathore, S., \& Rahulkar, A D. Design and implementation of PID controller based on orthogonal wavelet filter-banks in FPGA. 2017 7th International Symposium on Embedded Computing and System Design (ISED). doi:10.1109/ised.2017.8303928

12. Parvez, Shahid and Zhiqiang Gao, "A wavelet-based multiresolution PID controller", IEEE Transactions on Industry Applications, 2005, Vol.41, No.2, pp.537-543

13. Vajpayee, Vineet, Siddhartha Mukhopadhyay and AkhilanandPatiTiwari, "A Multi resolution Wavelet based Subspace Identification", An International Journal of IFAC ,2016, Vol.49, No.1, pp.247-253.

14. Dewan L and Garg M. , "A Numerical Method for Linear ODEs using Non-recursive Haar Connection Coefficients", International Journal of Computational and Applied Mathematics (IJCAM) vol. 2 no.3b,pp 429-440,2012.

15. Khan, M.Abdesh SK and M.AzizurRahman, "A novel neuro-wavelet-based self-tuned wavelet controller for IPM motor drives", IEEE Transactions on Industry Applications, 2010,Vol.46, No.3, pp.1194-1203.

16. Kanungo, A., Dewan, L., \& Mittal, M.," Performance improvement of a thermal system PID controller using Haar wavelet based denoising". 


\section{Optimal Parameter Tuning of MRPID Controller for Temperature Control}

International Conference on Industrial Instrumentation and Control (ICIC). 2015, doi:10.1109/iic.2015.7150773.

17. Khan, M. A. S. K., \& Rahman, M. A. "Implementation of a Wavelet-Based MRPID Controller for Benchmark Thermal System". IEEE Transactions on Industrial Electronics, 57(12), 2010 4160-4169.doi:10.1109/tie.2010.2044121

18. S. Parvez,, "Advanced control techniques for motion control problem",2003, Ph.D. dissertation, Cleveland State Univ., Cleveland.

19. Meena, D. C., \& Devanshu, A. "Genetic algorithm tuned PID controller for process control”. 2017 International Conference on Inventive Systems and Control (ICISC). doi:10.1109/icisc.2017.8068639.

20. Seekuka, J., Rattanawaorahirunkul, R., Sansri, S., Sangsuriyan, S., \& Prakonsant, A. "AGC using Particle Swarm Optimization based PID controller design for two area power system. "International Computer Science and Engineering Conference (ICSEC). doi:10.1109/icsec.2016.7859951.

21. Aranza, M. F., Kustija, J., Trisno, B., \& Hakim, D. L. "Tunning PID controller using particle swarm optimization algorithm on automatic voltage regulator system". IOP Conference Series: Materials Science and Engineering, 2016,128, 012038. doi:10.1088/1757-899x/128/1/012038

\section{AUTHORS PROFILE} Abhas Kanungo has obtained his Bachelor's degree in
Instrumentation and control Engineering from RGTU, Bhopal in 2008. Then he obtained his Master's degree in Control systems from National Institute of Technology, Kurukshetra, Haryana India in 2010 and now doing Ph.D. from National Institute of Technology, Kurukshetra, Haryana, India. His current research interests are Wavelet based control, signal processing. He is Assistant Professor in EIE Dept., KIET Group of Institutions, Ghaziabad from last 5.5 years.

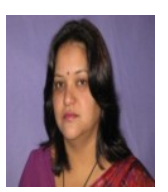

Dr. Monika Mittal has completed her Graduation in Electrical Engineering from M. M. M. Institute of Technology, Gorakhpur, India in 1992 and completed her Post Graduation from NIT Kurukshetra, India in 1994 with specialization in Control Systems. She has completed her doctorate from Electrical Engineering Dept., NIT Kurukshetra in 2013. She has a teaching experience of about 25 years. She has authored about 50 research papers in International and National journals and conferences. Presently, she is working in the areas of signal processing applications in control systems, computational algorithms, wavelets in control. Presently she is working on Post of Associate professor in Electrical engineering department NIT Kurukshetra.

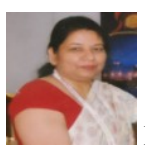

Dr. Lillie Dewan has completed her Graduation in Electrical engineering from Punjab university in 1983 and completed her Post Graduation from Kurkshetra university, Haryana, India in 1987 with specialization in Control. She has completed her doctorate in year 2001. She has a teaching experience of about 31 years. She has authored about 130 research papers in International and National journals and conferences. Presently, she is working in the areas of Control (Adaptive and Robust), Instrumentation, Biomedical signal Processing. Presently she is working on Post of professor in Electrical engineering department NIT Kurukshetra. 